\title{
Prevalencia de la enfermedad periodontal crónica en pacientes diabéticos.
}

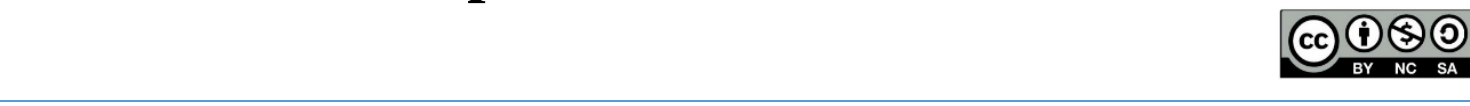

Periodontal disease chronic Diabetes in patient

Maritza Madan O’Farril. ${ }^{1}$, Lázaro Gasca Martin. ${ }^{2}$, Suleidys Espasandin González. ${ }^{3}$ \& Efraín Velasteguí López. ${ }^{4}$

\section{DOI: https://doi.org/10.33262/anatomiadigital.v4i4.1891}

\begin{abstract}
.
Resumen.

A descriptive, cross-sectional Se realizó un estudio observacional observational study was carried out in the descriptivo de corte transversal en la stomatological clinic of the Güines clínica estomatológica del municipio municipality, Mayabeque province, from September-2018 to May-2020 to Güines, provincia Mayabeque, de septiembre-2018 a mayo-2020 para determine the prevalence and severity of chronic periodontitis in patients with Diabetes Mellitus. The study population consisted of 126 patients over 16 who attended the dental clinic with the disease. The information was collected determinar la prevalencia y severidad de la periodontitis crónica en los pacientes con Diabetes Mellitus. La población objeto de estudio estuvo constituida por los 126 pacientes mayores de 16 que acudieron a la clínica estomatológica

\footnotetext{
1 Facultad de Ciencias Médicas Mayabeque, Clínica Estomatológica Docente “Andrés Ortiz Junco". Güines, Cuba. Dhttps://orcid.org/0000-0002-9205-6970. Correo electrónico: maritzamo@infomed.sld.cu;.

2 Facultad de Ciencias Médicas Mayabeque, Clínica Estomatológica Docente “Andrés Ortiz Junco". Güines, Cuba. Correo electrónico:lazaro@nauta.com

3 Facultad de Ciencias Médicas Mayabeque, Clínica Estomatológica Docente "Andrés Ortiz Junco". Güines, Cuba. correo electrónico Sespasandin@infomed.sld.cu

${ }^{4}$ Universidad técnica de Babahoyo, Babahoyo Los Ríos, evelasteguil@utb.edu.ec
} 
through a spreadsheet prepared for this purpose and processed through a database in electronic format, using descriptive statistics techniques. It was concluded that more than half of the examined population was between 16 and 30 years old (57.1), belonged to the female sex $(61.9 \%)$, the majority suffered from periodontitis (40.5\%), from Type II Diabetes Mellitus (83.3\%) with more than 5 years of evolution (76.2\%) and little stability in glycemic values $(66.7 \%)$.

Key words: Risk Factors, Periodontitis, Diabetes Mellitus. portadores de la enfermedad. La información se recogió a través de una planilla confeccionada para tal efecto y se procesó mediante una base de datos en formato electrónico, utilizándose técnicas de estadísticas descriptivas. Se concluyó que más de la mitad de la población examinada tenía entre 16 y 30 años (57.1), pertenecía al sexo femenino (61.9\%), la mayoría padecía de periodontitis $(40.5 \%)$, de Diabetes Mellitus Tipo II $(83.3 \%)$ con más de 5 años de evolución (76.2\%) y poca estabilidad en los valores de glucemia $(66.7 \%)$.

Palabras claves: factores de riesgos, diabetes y enfermedad periodontal.

\section{Introducción}

La enfermedad periodontal (EP) es un trastorno inflamatorio crónico complejo causado por la infección de los tejidos de soporte que rodean a los dientes. La infección comienza con la colonización y crecimiento de un pequeño grupo de espiroquetas y bacterias predominantemente Gram-negativas anaeróbicas (Botero et. al.;2008). Estas bacterias mezcladas con numerosas especies del "biofilm" bacteriano también llamado placa dentaria se extienden apicalmente a lo largo de la superficie de la raíz dentaria provocando la formación de bolsas periodontales, la destrucción del hueso alveolar y la desinserción de las fibras de colágeno del ligamento periodontal, lo que acaba produciendo pérdida del diente. Generalmente el diagnóstico clínico de la periodontitis se basa en cuantificar la presencia y extensión de las bolsas periodontales, la pérdida de inserción clínica, el patrón y la extensión de la pérdida de hueso alveolar valorado radiográficamente, o una combinación de estas medidas (Aristazabal;2012).

La prevalencia de la EP severa, que lleva a la pérdida de hueso es muy alta, afectando aproximadamente del 10\% al 15\% de los adultos a nivel mundial de acuerdo con la OMS (Guilarte; 2015). La EP moderada es mucho más frecuente, afectando al 40-60\% de los adultos, aunque estas cifras varían según los criterios diagnósticos utilizados y según los grupos sociales, etnias, estado socioeconómico e higiénico etc. Por todo ello la EP se considera un problema prevalente e importante en cuanto que, sin ser mortal, genera problemas de salud tanto oral como general y deterioro de la calidad de vida, aunque muchas veces los sistemas sanitarios no le prestan atención (Guilarte; 2011).

La asociación entre infecciones microbianas orales como las periodontopatías y los desórdenes sistémicos no es un concepto nuevo; fue planteada por primera vez por los 
sirios en el siglo VII a.c. Según datos aportados por Genco, estas afectan aproximadamente al $18 \%$ de la población comprendida entre 25 y 30 años, con franca tendencia al incremento de su incidencia con la edad, siendo la principal causa de pérdida dental en individuos adultos (Farias; 2012).

En Cuba aproximadamente el 16\% de los niños entre los 8 y 11 años con diabetes mellitus tiene periodontitis y los jóvenes adultos insulinodependientes muestran un incremento significativo en la destrucción de los tejidos periodontales comparado con personas sin diabetes. También se ha demostrado la relación entre las periodontopatías y las diferentes enfermedades sistémicas. Donde más de $70 \%$ de la población adulta está afectada con enfermedades periodontales a partir de los 30 años, y más de un $15 \%$ se ha tratado con dicha patología, en muchos casos debido a enfermedades sistémicas como la Diabetes Mellitus.

En los últimos años el estado de salud de la población cubana, particularmente la salud bucal, ha experimentado notables avances, como resultado de la alta prioridad que se ha brindado a aquellos aspectos básicos y determinantes de la calidad de vida de los ciudadanos (Safkan; 2015).

No obstante, existe una alta prevalencia y severidad de las manifestaciones clínicas de la EP crónica en los pacientes diabéticos que asisten a la consulta.

Por lo que es importante indagar en los factores de riesgos que están causando la enfermedad periodontal en los pacientes de la clínica estomatológica Andrés Ortiz, donde hasta el momento no existen datos al respecto, ya que este tipo de estudio permitirá obtener resultados estadísticos que serán de mucha importancia para que los especialistas en salud bucal a nivel local y nacional conozcan los factores de riesgos que la provocan, su prevalencia, severidad y localización, lo que hará posible el diseño de estrategias de salud bucal orientadas a promover la prevención, tratamiento y control de la enfermedad periodontal, contribuyendo a que se desarrolle la calidad de vida de los individuos afectados.

\section{Metodología}

Se realizó una investigación observacional descriptiva de corte transversal. en el período de tiempo comprendido de septiembre del 2018 a mayo de 2020 con el objetivo de determinar la prevalencia y severidad de la periodontitis crónica en los pacientes con Diabetes Mellitus. El universo estuvo conformado por todos los pacientes mayores de 16 años diabéticos que fueron atendidos en la consulta de periodoncia de la clínica estomatológica de Güines., se utilizaron las variables, edad, sexo, gravedad de la enfermedad periodontal, tipo de diabetes mellitus, tiempo de diagnosticada y estabilidad de los valores de glucemia La información recogida en la planilla se procesó mediante la creación de una base de datos en formato electrónico, Microsoft Access.

Para el análisis de los datos se emplearon técnicas de estadísticas descriptivas y se determinó las frecuencias absolutas de cada variable estudiada reflejadas en tablas de 
contingencia 2x2 a través de la dócima $\mathrm{X}^{2}$ (Steel and Torrie; 1988, Duncan;1955)

Para el procesamiento de la información se utilizó el software estadístico InfoStat 2012 y ComparPro para el análisis de Duncan, la edición del trabajo se realizó con el uso de Microsoft Excel y Word.

Los resultados se presentarán en tablas y se utilizará el porciento como unidad resumen. Se analizará cada tabla, y se realizarán comparaciones con los hallazgos de otros autores sobre la base de un análisis inductivo y deductivo que permitirá dar salida a los objetivos propuestos, y así se emitirán conclusiones y recomendaciones

\section{Resultados}

Al analizar la distribución porcentual de pacientes diabéticos según edad y sexo se evidencia que el grupo de edades más afectado resultó ser de 16 a 30 años con un $57,1 \%$ seguido del 31 a 45 con 16,2\% y el de 46 a 60 años con un 11,9\% en cada uno de ellos con diferencias significativas entre ellos. En cuanto al sexo predominó el sexo femenino con un $61,9 \%$ con diferencias significativas respecto al masculino que se presentó con un $38,1 \%$.

Según criterios del autor, estos resultados pudieran atribuirse a que las mujeres por tener una mayor preocupación por su estética y por su salud bucal, acuden a consulta con mayor frecuencia que los hombres. Además, se ha demostrado en numeroso estudio, que existen estados funcionales como el periodo menstrual, el embarazo y la menopausia que influyen en el curso de esta enfermedad y por tanto, hacen a las féminas más susceptibles. Respecto a la edad, el predominio de las periodontopatías en la población más joven, pudiera estar determinada por el consumo desmedido de una dieta rica en carbohidratos a modo de meriendas y comidas rápidas, que resulta tan frecuente, en el ámbito estudiantil y laboral, y que constituye sin dudas, un riesgo para la aparición de enfermedades crónicas.

Estos resultados coinciden con otros autores como (Pérez et. al., 2015) quienes también encontraron un predominio de la enfermedad periodontal en pacientes jóvenes y del sexo femenino, y difieren con otras investigaciones (Herrera, 2017), donde se reporta más del $50 \%$ de pacientes masculinos y con edades entre 38 a 48 años.

Tabla \# 1: Distribución porcentual de los pacientes diabéticos según grupos de edades y sexo. Güines 2020.

\begin{tabular}{|c|c|c|c|c|c|c|}
\hline \multirow{3}{*}{$\begin{array}{l}\text { Grupos } \\
\text { De } \\
\text { Edades }\end{array}$} & \multicolumn{4}{|c|}{ Sexo } & & \\
\hline & \multicolumn{2}{|c|}{ Femenino } & \multicolumn{2}{|c|}{ Masculino } & \multicolumn{2}{|c|}{ Total } \\
\hline & No & $\%$ & No & $\%$ & No & $\%$ \\
\hline $16-30$ & 48 & $38.1 \mathrm{a}$ & 24 & $19.0 b$ & 72 & $57.1 \mathrm{a}$ \\
\hline $31-45$ & 21 & $16.7 \mathrm{bc}$ & 12 & $9.5 c$ & 33 & $16.2 b$ \\
\hline $46-60$ & 3 & $2.4 d$ & 12 & $9.5 c$ & 15 & $11.9 \mathrm{c}$ \\
\hline 61- +años & 6 & $4.8 d$ & 0 & $0.0 \mathrm{~d}$ & 6 & $4.8 \mathrm{c}$ \\
\hline EE y Sign & \multicolumn{4}{|c|}{ $\pm 2.9^{\star * *}$} & \multicolumn{2}{|c|}{ \pm 3.9 ** } \\
\hline Total & 78 & $61.9 a$ & 48 & $38.1 \mathrm{~b}$ & 126 & 100.0 \\
\hline EE y Sign & \multicolumn{4}{|c|}{ $\pm 4.4^{* \star *}$} & & \\
\hline
\end{tabular}

Fuente: Elaboración propia. 
$\mathrm{Al}$ analizar en la tabla 2, la severidad de la enfermedad se observa un predominio de la periodontitis con un $40,5 \%$ con diferencias significativas con la periodontitis severa y la gingivitis con $28,8 \%$ y $26,2 \%$ respectivamente. Es necesario aclarar que ningún paciente presentó la encía sana por lo que no se colocó en la tabla dicho parámetro.

Según Coke (2012), las personas con diabetes deben ser evaluadas por su médico antes de coordinar un tratamiento para la enfermedad periodontal, ya que, los problemas periodontales pueden complicar el control de la diabetes Mellitus, y una diabetes descontrolada puede agravar la enfermedad periodontal, planteamiento que justifica que la totalidad de la población examinada haya tenido algún signo de enfermedad periodontal.

Los resultados coinciden con los obtenidos por Quisinina (2012), se refiere que el 81\% de los pacientes diabéticos tipo II durante el examen bucodental fueron diagnosticados con enfermedad periodontal, teniendo el mayor porcentaje la periodontitis leve con un $71 \%$.

Tabla \# 2: Distribución porcentual de pacientes diabéticos según severidad de la enfermedad periodontal por el índice de Russell. Güines 2020

\begin{tabular}{lcc}
\hline Gravedad de la enfermedad periodontal & No & $\%$ \\
Gingivitis leve & 6 & $4.8 \mathrm{c}$ \\
Gingivitis & 33 & $26.2 \mathrm{~b}$ \\
Periodontitis & 51 & $40.5^{\mathrm{a}}$ \\
Periodontitis severa & 36 & $28.6 \mathrm{ab}$ \\
EE y Sign & \multicolumn{2}{c}{ $\pm 3.6^{\star * *}$} \\
Total & $126^{2}$ & 100 \\
\hline
\end{tabular}

Fuente: Elaboración propia

En la tabla 3 se observa un mayor predominio de pacientes con Diabetes Mellitus tipo II para un $83,3 \%$, siendo el grupo más representativo el que incluye los pacientes con periodontitis para un $33,3 \%$, lo cual evidencia diferencias altamente significativas respecto al grupo de diabéticos tipo I representado por el $16,7 \%$.

Al relacionar el tipo de Diabetes Mellitus y la severidad de la enfermedad periodontal, la tabla evidencia que los más afectados son los diabéticos Tipo II, lo cual pudiera atribuirse a que estos representan el grupo más numeroso, pero, además, demuestra que un control metabólico inadecuado incrementa la severidad de la enfermedad periodontal y, por tanto, la necesidad de tratamientos más complejos.

Varios estudios coinciden con los resultados obtenidos, algunos demuestran que la prevalencia de la diabetes tipo II está aumentando a un ritmo alarmante, debido principalmente, a un estilo de vida sedentario y al incremento de la obesidad. Dado que la diabetes tipo II representa aproximadamente el $90 \%$ de todos los casos de diabetes, esta afección está alcanzando rápidamente proporciones epidémicas (Cabrera; 2018).

Otro estudio de Quisinina (2012), demuestra que en personas con diabetes mellitus dependientes de insulina, la enfermedad periodontal es más severa y más prevalente que 
en diabéticos no dependientes y en un estudio longitudinal realizado por (Ramírez et. al., 2009) en pacientes con diabetes Mellitus de tipo II, los que padecían enfermedad periodontal avanzada presentaron un estado diabético significativamente peor que los que tenían enfermedad periodontal mínima.

Estudios de Ramírez et. al. (2009), reportan que la incidencia de la Diabetes Mellitus tipo II, es de 8 por cada 1.000 habitantes al año.

Tabla \# 3: Distribución porcentual de pacientes diabéticos según severidad de la enfermedad periodontal y tipos de Diabetes Mellitus. Güines 2020

\begin{tabular}{|c|c|c|c|c|c|c|}
\hline \multirow{3}{*}{$\begin{array}{l}\text { Gravedad de la } \\
\text { Enfermedad } \\
\text { Periodontal }\end{array}$} & \multicolumn{4}{|c|}{ Tipos de diabetes Mellitus } & & \\
\hline & \multicolumn{2}{|c|}{ Tipo I } & \multicolumn{2}{|c|}{ Tipo II } & \multicolumn{2}{|c|}{ Total } \\
\hline & No & $\%$ & No & $\%$ & No & $\%$ \\
\hline Gingivitis leve & 3 & 2.4 & 3 & 2.4 & 6 & $4.8 \mathrm{c}$ \\
\hline Gingivitis & 3 & 2.4 & 30 & 23.8 & 33 & $26.2 \mathrm{~b}$ \\
\hline Periodontitis & 9 & 7.1 & 42 & 33.3 & 51 & $40.5 a$ \\
\hline $\begin{array}{l}\text { Periodontitis } \\
\text { severa }\end{array}$ & 6 & 4.8 & 30 & 23.8 & 36 & $28.6 \mathrm{~b}$ \\
\hline EE y Sign & \multicolumn{4}{|c|}{ $\pm 2.9 \mathrm{NS}$} & \multicolumn{2}{|c|}{ \pm 3.9 *** } \\
\hline Total & 21 & $16.7 b$ & 105 & $83.3^{\mathrm{a}}$ & 126 & 100.0 \\
\hline EE y Sign & \multicolumn{4}{|c|}{ $\pm 4.4^{* \star *}$} & & \\
\hline
\end{tabular}

En la tabla 4 se observa que el 76,2 \% de los pacientes, tienen un tiempo de evolución de más de 5 años, predominando la periodontitis con un $31 \%$, con diferencias significativas respecto a los de menos de 5 años, representados solo por el $23,8 \%$.

Como se aprecia, la afectación periodontal es directamente proporcional al tiempo de diagnosticada la Diabetes Mellitus, lo cual puede atribuirse a que durante el curso de esta enfermedad ocurre un retardo en la formación de linfocitos y disminución de su activación, además disminuye el aporte sanguíneo del periodonto por la microangiopatía diabética, donde los vasos sanguíneos, por alteración de sus capas internas, disminuyen su luz y ello trae como consecuencia la disminución del transporte de los nutrientes y el oxígeno. Por otra parte, el periodonto se hace más vulnerable a los embates del medio externo por disminución de la función de los polimorfo nucleares neutrófilos.

La síntesis del colágeno parece estar afectada por los niveles de glucosa, además que los fibroblastos gingivales sintetizan menos colágeno que en sujetos no diabéticos, también se ha notado un aumento en la actividad de la enzima colagenasa en los tejidos gingivales y de la actividad colagenolítica del fluído gingival.

Rodríguez et. al. (2015), también es del criterio que el estado periodontal de los pacientes está relacionado con el tiempo de padecimiento de la diabetes mellitus, ya que pudo observar que a medida que avanza el tiempo de padecimiento de la diabetes mellitus, aumentaba la condición clínica de la enfermedad periodontal. 
Tabla \# 4: Distribución porcentual de pacientes diabéticos según el tiempo de diagnóstico de Diabetes Mellitus. Güines 2020

\begin{tabular}{|c|c|c|c|c|c|c|}
\hline \multirow{3}{*}{$\begin{array}{l}\text { Gravedad de la } \\
\text { enfermedad } \\
\text { Periodontal }\end{array}$} & \multicolumn{4}{|c|}{ Tiempo de diagnóstico de la DM } & & \\
\hline & \multicolumn{2}{|c|}{ Menor de 5 años } & \multicolumn{2}{|c|}{ Mayor de 5 años } & \multicolumn{2}{|c|}{ Total } \\
\hline & No & $\%$ & No & $\%$ & No & $\%$ \\
\hline Encía sana & - & - & - & - & - & - \\
\hline Gingivitis leve & 6 & $4.8 \mathrm{~cd}$ & 0 & $0.0 \mathrm{~d}$ & 6 & $4.8 \mathrm{c}$ \\
\hline Gingivitis & 9 & $7.1 \mathrm{~cd}$ & 24 & $19.1 b$ & 33 & $26.2 \mathrm{~b}$ \\
\hline Periodontitis & 12 & $9.5 c$ & 39 & $31.0 \mathrm{a}$ & 51 & $40.5 \mathrm{a}$ \\
\hline $\begin{array}{l}\text { Periodontitis } \\
\text { severa }\end{array}$ & 3 & $2.4 \mathrm{~cd}$ & 33 & $26.2 \mathrm{a}$ & 26 & $28.6 \mathrm{~b}$ \\
\hline EE y Sign & \multicolumn{4}{|c|}{ $\pm 2.9^{\star * *}$} & \multicolumn{2}{|c|}{ $\pm 3.9^{* \star \star}$} \\
\hline Total & 30 & $23.8 \mathrm{~b}$ & 96 & $76.2 \mathrm{a}$ & 126 & 100.0 \\
\hline EE y Sign & \multicolumn{4}{|c|}{ $\pm 4.4^{* * *}$} & & \\
\hline
\end{tabular}

Fuente: Elaboración propia

Se observa en la tabla 5 que el $66,7 \%$ de los pacientes en el estudio no presentaba estabilidad en los valores de glucemia, con diferencias significativas respecto a los que mantienen compensado su estado sistémico para un 33,3\%, lo que justifica el hecho de que la totalidad de la población estudiada estaba afectada desde el punto de vista periodontal.

Estos resultados son consecuencia de la descompensación metabólica, la cual produce acumulación de los productos avanzados de glucosilación (PAG) no enzimática, lo que afecta la migración y la fagocitosis, tanto de los polimorfonucleares como de los mononucleares, lo que favorece el establecimiento de una flora subgingival que por maduración se irá transformando en flora predominantemente anaerobia gram negativa. Lo señalado anteriormente determina el aumento de la secreción de mediadores inflamatorios que provocarán la destrucción del tejido conectivo, la reabsorción ósea y el desarrollo de una resistencia a la insulina en el tejido. Además, la enfermedad periodontal severa puede tener malos efectos en el control de la diabetes, pues al tener molestias, la persona con diabetes puede decidir comer alimentos que son más fáciles de masticar, pero que pueden ser no apropiados para su plan de alimentación y que no garanticen un buen control metabólico.

Estos resultados son similares a los expresados por Cabrera (2018), que plantea que en las personas con diabetes Mellitus y mal control metabólico, es favorable el avance de la EP.

Tabla \# 5: Distribución porcentual de pacientes diabéticos según severidad de la enfermedad periodontal y estabilidad en los valores de glucemia. Güines 2020

\begin{tabular}{|c|c|c|c|c|c|c|}
\hline \multirow{3}{*}{$\begin{array}{c}\text { Gravedad de la } \\
\text { enfermedad } \\
\text { Periodontal }\end{array}$} & \multicolumn{4}{|c|}{ Estabilidad en los valores de glucemia. } & \multirow{2}{*}{\multicolumn{2}{|c|}{ Total }} \\
\hline & \multicolumn{2}{|c|}{ Estable } & \multicolumn{2}{|c|}{ No estable } & & \\
\hline & No & $\%$ & No & $\%$ & No & $\%$ \\
\hline Encía sana & - & - & - & - & - & - \\
\hline Gingivitis leve & 6 & $4.8 \mathrm{~d}$ & 0 & $0.0 \mathrm{~d}$ & 6 & $4.8 \mathrm{c}$ \\
\hline Gingivitis & 12 & $9.5 \mathrm{c}$ & 21 & $16.7 \mathrm{~b}$ & 33 & $26.2 b$ \\
\hline Periodontitis & 21 & $16.7 \mathrm{~b}$ & 30 & $23.8 \mathrm{a}$ & 51 & $40.5 a$ \\
\hline
\end{tabular}


Tabla \# 5: Distribución porcentual de pacientes diabéticos según severidad de la enfermedad periodontal y estabilidad en los valores de glucemia. Güines 2020 (continuación)

\begin{tabular}{|c|c|c|c|c|c|c|}
\hline \multirow{3}{*}{$\begin{array}{l}\text { Gravedad de la } \\
\text { enfermedad } \\
\text { Periodontal }\end{array}$} & \multicolumn{6}{|c|}{ Estabilidad en los valores de glucemia. } \\
\hline & \multicolumn{2}{|c|}{ Estable } & \multicolumn{2}{|c|}{ No estable } & \multicolumn{2}{|c|}{ Estable } \\
\hline & No & $\%$ & No & $\%$ & No & $\%$ \\
\hline $\begin{array}{l}\text { Periodontitis } \\
\text { severa }\end{array}$ & 3 & $2.4 \mathrm{~d}$ & 33 & $26.2 a$ & 36 & $28.6 \mathrm{~b}$ \\
\hline EE y Sign & \multicolumn{4}{|c|}{ $\pm 2.9^{\star \star *}$} & \multicolumn{2}{|c|}{ $\pm 3.9^{* \star *}$} \\
\hline Total & 42 & $33.3 b$ & 84 & $66.7 \mathrm{a}$ & 126 & 100.0 \\
\hline EE y Sign & & & & & & \\
\hline
\end{tabular}

Fuente: Elaboración propia

\section{Conclusiones}

- De los pacientes examinados, más de la mitad pertenecía al grupo de 16 y 30 años y al sexo femenino.

- Prevalecieron los pacientes afectados por periodontitis crónica leve.

- Predominaron los diagnosticados con Diabetes Mellitus Tipo II de más de 5 años de evolución.

- La mayoría de los pacientes presentaron poca estabilidad en los valores de glucemia.

\section{Referencias Bibliográficas}

Aristizabal, J (2012). Sensibilidad a la Amoxicilina de bacterias anaerobias de pacientes con periodontitis agresiva. Reviste CES Odontología. Vol. 25. No. 1.

Bascones A, Caballeros A. Actinobacillus Actinomycetemcomitans y Porphyromonas Gingivales como principales patógenos periodontales. Avances en Periodoncia. 2014 Sep.; 12(2): 69-75.46.

Botero, A; Alvear, F.S; Vélez, M.E; Botero, L; Velásquez, H (2008). Evaluación de los enfoques terapéuticos para las varias formas de enfermedad periodontal. Parte III: Prevalencia de Bacilos entéricos y levaduras. Revista Facultad Odontología Universidad Antioquia; 20(1): 72-86.

Cabrera, E; Licea, M.E (2018). Nueva definición, prevalencia, caracterización y tratamiento de la diabetes autoinmune latente del adulto. Rev Cubana Endocrinol. 19(3). Disponible en: http://scielo.sld.cu/scielo.php?script=sci_arttext\&pid=S15612953200800030000 $8 \& \operatorname{lng}=$ es\&nrm=iso\&tlng=es

Coke, K (2012). Enfermedad periodontal en paciente diabético. Disponible en: http://html.rincondelvago.com/enfermedad-periodontal-en-pacientediabetico.html. 
Di Rienzo, J; Balzarini, M; Casanoves, F; Gonzále, L; Tablada, M; Guzmán, W y Robledo, C.R (2001). InfoStat. Software estadístico. Universidad Nacional de Argentina.

Duncan, D.B (1955). Multiple range and multiple F test. Biometrics 11(1).

Farias, F (2012). Enfermedad periodontal y microorganismos periodonto patógenos. Revista de facultad de Odontología. Universidad de Maracaibo.

Guilarte, C; Perrone, M (2015). Bacterias Periodonto patógenas: Bacilos Anaerobios gran negativos como agentes Etiológicos de la Enfermedad Periodontal. Acta odontol.venez. Mayo.

Guilarte, C (2011). Patógenos Periodontales: Revisión de literatura. Acta odontología. Venezuela. Dic; 39(3): 91-93.

Herrera, CA (2017). Prevalencia de periodontitis crónica generalizada en pacientes de la clínica odontológica de la UDLA en el periodo de enero del 2012 a 2017. Disponible en: http://sisbib.unmsm.edu.pe/bibvirtualdata/tesis/salud/allende_rp/clasificacion.pd $\mathrm{f}$

Pérez, Y; Díaz, A; Navarrete, J; Curbelo Y (2015). Incidencia de diabetes mellitus tipo 1 en la provincia Mayabeque. Medimay. Disponible en: http://revcmhabana.sld.cu/index.php/rcmh/article/view/754

Quisinina, N (2012). Relación de la enfermedad periodontal en pacientes diabéticos tipo 2, del club de diabéticos en el Hospital IESS Riobamba a partir de diciembre/2011, hasta agosto/2012. Disponible en: http://www.intramed.net/contenidover.asp?contenidoID=72517

Ramírez, E; Álvarez, D; García, R; Álvarez, M; Rodríguez, Y; Matos, Y (2009). Diabetes mellitus en Ciego de Ávila: serie secular 1997-2008. Revista Cubana Endocrinol. 20(3). Disponible en: http://scielo.sld.cu/scielo.php?script=sci_arttext\&pid=S15612953200900030000 $4 \& \operatorname{lng}=$ es\&nrm=iso

Rodríguez, Y; Alemán, E; Rodríguez, R; Valdivia, MI; Galá, E; Díaz, G (2015). Enfermedad periodontal inmunoinflamatoria crónica en pacientes diabéticos en edad pediátrica. Rev $\mathrm{Cub}$ de Estomatol. 52. Disponible en: http://www.scielo.cl/scielo.php?pid=s071901072012000200008\&script=sci_artt ext

Safkan, A.J(2015). Periodontal conditions ininsulin-dependent diabetes mellitus. J Clin Periodontol; 19:24-29

Steel, R.G and Torrie, I.H (1988). Bioestadística principios y procedimientos. McGrawHill. Interamericana. Mexico S. A. 740 pp. 


\section{PARA CITAR EL ARTÍCULO INDEXADO.}

Madan O’Farril, M., Gasca Martin, L., Espasandin González, S., \& Velasteguí López, E. (2021). Prevalencia de la enfermedad periodontal crónica en pacientes diabéticos. Anatomía Digital, 4(4), 69-78. https://doi.org/10.33262/anatomiadigital.v4i4.1891

\section{Liencia}

El artículo que se publica es de exclusiva responsabilidad de los autores y no necesariamente reflejan el pensamiento de la Revista Anatomía Digital.

El artículo queda en propiedad de la revista y, por tanto, su publicación parcial y/o total en otro medio tiene que ser autorizado por el director de la Revista Anatomía Digital.
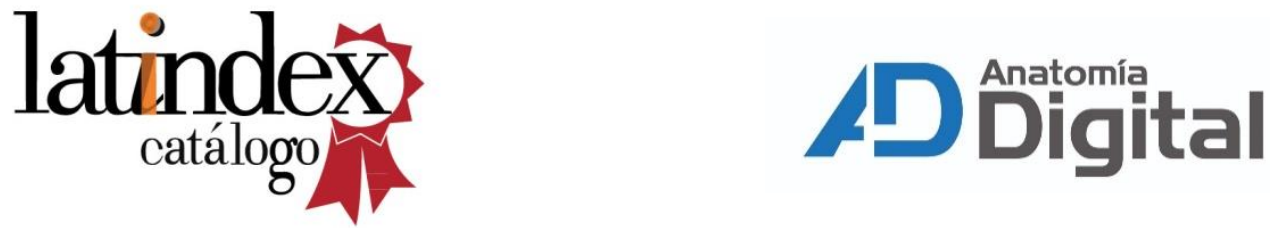